\title{
Alfabetización, razonamiento y pensamiento estadísticos: competencias específicas que requieren promoverse en el aula
}

\author{
Statistical literacy, reasoning and thinking: Specific competences that need to be promoted in classroom
}

\author{
Eduardo Aguilar Fernández \\ José Andrey Zamora Araya \\ Helen Susana Guillén Oviedo
}

\begin{abstract}
RESUMEN
El objetivo principal del ensayo consiste en analizar cómo el enfoque por competencias puede contribuir al cumplimiento de los fines de la educación estadística. Se realiza una revisión sobre el concepto de competencias, sus tipos y enfoque. Posteriormente se analiza la relación entre el enfoque por competencias y la educación estadística, de tal manera que la incorporación de la alfabetización, razonamiento y pensamiento estadístico se consideren como competencias por desarrollar. Asimismo se brindan definiciones de competencias matemáticas y estadísticas, enfatizando en que son diferentes pero complementarias. Finalmente se rescatan las recomendaciones del informe de la Guidelines for Assessment and Instruction in Statistics Education (GAISE) para la enseñanza de la estadística y sugerencias de cómo podrían implementarse las competencias estadísticas en las aulas bajo un enfoque por competencias.
\end{abstract}

Palabras claves: competencias estadísticas, competencias matemáticas, educación estadística, enfoque por competencias.

\section{ABSTRACT}

The main objective of this essay is to analyze how the competencies approach can contribute to fulfilling the goals of statistics education. A revision about the concept of competencies, their types and approach is performed. Additionally, the relationship between competencies and Statistics education, along with the way to incorporate literacy, reasoning, and statistical thinking as competencies to be developed in class is analyzed. Besides, definitions are given about mathematics and statistics competencies emphasizing that although they complement each other, are different. Finally, recommendations and suggestions of the Guidelines for Assessment and Instruction in Statistics Education (GAISE) report are given for teaching statistics and how they could be applied in the classrooms under a competencies approach.

Keywords: statistics competencies, mathematics competencies, statistics education, competencies approach. 


\section{INTRODUCCIÓN}

Con el paso de los años, varios cambios se han sugerido en los procesos de enseñanza y aprendizaje, entre ellos los que promueven el paso de métodos tradicionales a metodologías más participativas, en las que el estudiantado se convierta en un ente más activo en su proceso de formación. Una de las estrategias planteadas para este cambio es la incursión de planes de estudios basados en el enfoque por competencias, esto con el fin de promover en las personas el desarrollo de habilidades que les permitan adaptarse a las continuas transformaciones que la sociedad actual experimenta.

Por otro lado, el creciente número de estudios relacionados con la forma en que se imparten los cursos de Estadística en los distintos niveles educativos han expuesto, entre otras cosas, la relevancia de algunos términos, la aparición de nuevos conceptos, las dificultades que enfrentan las personas cuando aprenden estadística, la discusión sobre la necesidad de revisar los métodos tradicionales de enseñanza, así como la de ofrecer orientaciones más específicas a las personas que enseñan estadística.

El objetivo principal del estudio consiste en analizar cómo el enfoque por competencias puede contribuir al cumplimiento de los fines de la educación estadística y además resaltar la diferencia que existe entre competencias matemáticas y estadísticas.

Para alcanzar este objetivo, primero se enumeran algunas de las concepciones que se han asociado al término "competencias", con el fin de tener una visión inicial sobre su significado, así como para identificar algunos de los elementos que son comunes. Además se detallan aspectos que caracterizan la metodología de enseñanza basada en el enfoque por competencias y algunos de los argumentos que justifican su inclusión en los programas educativos; asimismo se exponen aspectos relacionados con la educación estadística. Finalmente se identifican la concepciones de competencia matemática y estadística y cómo estas pueden considerarse en el área de la estadística bajo el enfoque por competencias.

Eduardo Aguilar Fernández. Profesor-investigador de la Escuela de Matemática de la Universidad Nacional, Costa Rica. Es máster en Estadística por la Universidad de Costa Rica y cuenta con licenciatura en Enseñanza de la Matemática por la Universidad Nacional. Entre sus publicaciones recientes se encuentra el libro Introducción a la estadística descriptiva con $\mathrm{R}$ y el artículo "Correlación entre factores de riesgo de enfermedad cardiovascular en 1084 parejas de cónyuges costarricenses. El Proyecto Creless-RC” (2021). Correo electrónico: eduardo.aguilar.fernandez@una.cr. ID: https:/ /orcid.org/0000-0002-7864-2391.

José Andrey Zamora Araya. Profesor-investigador de la Escuela de Matemática de la Universidad Nacional, Costa Rica, y profesor de la Escuela de Estadística de la Universidad de Costa Rica. Cuenta con una maestría académica en Estadística y una maestría profesional en Economía del Desarrollo. Entre sus publicaciones recientes se encuentra el libro Introducción a la estadística descriptiva con R. Correo electrónico: jzamo@una.ac.cr. ID: https:/ /orcid.org/0000-0001-6050-5850.

Helen Susana Guillén Oviedo. Profesora-investigadora de la Escuela de Matemática de la Universidad Nacional, Costa Rica. Cuenta con una maestría en Matemática con mención en Estadística por la Universidad del Bío Bío (Chile). Su publicación más reciente es "Some aspects on the computational implementation of diverse terms arising in mixed virtual element formulations", en Numerical Algorithms (2021). Correo electrónico: hellen.guillen.oviedo@una.ac.cr. ID: https://orcid.org/0000-0002-5996-7772. 


\section{Concepto de competencia}

El término "competencia" ha derivado distintas definiciones según el ámbito en que se desarrolle, sea este laboral, económico o educativo. Uno de los primeros usos del término se remonta a los trabajos de Platón, que usa la palabra ikanótis para referirse a la cualidad de ser ikanos, traducido como capaz, tener la habilidad de conseguir algo, o destreza (Mulder, Weigel y Collins, 2007). Para la década de 1970, Chomsky propone el concepto de "competencia lingüística", considerándola como una estructura mental propia y determinada genéticamente que es aplicada cuando se llevan a cabo procesos de comunicación (Tobón, 2006).

A partir de este momento el concepto de competencias comienza a desarrollarse, trayendo consigo críticas y nuevas formulaciones en las que se hace referencia a la interacción de la persona con el entorno. Por ejemplo, Mejía (2010) define competencia como "un conjunto de potencialidades que posibilita un desempeño exitoso, que se materializa al responder a una demanda compleja que implica resolver un(os) problema(s) en un contexto particular, pertinente y no rutinario" (p. 1).

En el ámbito educativo se han propuesto varias definiciones al concepto, entre ellas la del proyecto Tuning, que entiende la competencia como "una combinación dinámica de atributos, en relación con conocimientos, habilidades, actitudes y responsabilidades, que describen los resultados de los aprendizajes de un programa educativo o lo que los estudiantes son capaces de demostrar al final del proceso educativo” (González y Wagenaar, 2003, p. 280). Asimismo, para Perrenoud (2004, p. 11) la competencia "representa una capacidad de movilizar varios recursos cognitivos para hacer frente a un tipo de situaciones".

Otra caracterización del término es la brindada por el Programme for International Student Assessment (PISA) de la Organización para la Cooperación y el Desarrollo Económicos (OCDE), para la cual la competencia “implica la capacidad de responder a demandas complejas, utilizando y movilizando recursos psicosociales (incluyendo habilidades y actitudes) en un contexto particular" (OCDE, 2004, p. 4).

Por su parte, la Asociación Nacional de Universidades e Instituciones de Educación Superior (ANUIES) expresa que la competencia "constituye un conjunto de conocimientos, habilidades y destrezas, tanto específicas como transversales, que debe reunir un titulado para satisfacer las exigencias sociales" (De Allende y Morones, 2006, p. 5). Por su parte, Tobón (2006, p. 5) menciona que "las competencias son procesos complejos de desempeño con idoneidad en un determinado contexto, con responsabilidad".

Asimismo es posible encontrar muchas otras formas de clasificar, interpretar y definir el concepto de competencias. Al respecto Weimer (2001, citado por Mulder Mulder, Weigel y Collins, 2007) menciona nueve: habilidad cognitiva general, des- 
trezas cognitivas especializadas, modelo de competencia-desempeño, modelo de competencia-desempeño modificado, tendencias de acción motivada, autoconcepto objetivo y subjetivo, competencia en acción, competencias claves y meta-competencias.

Las definiciones anteriores concuerdan en que las competencias involucran capacidades de la persona que favorecen el desarrollo de diferentes acciones que permitan enfrentar una situación específica. Es decir, se es competente si al presentarse un escenario particular no solamente se cuenta con los recursos cognitivos, materiales o de otra índole, sino que además las personas son capaces de utilizarlos para hacerle frente a las tareas que son demandadas. Por otro lado, estas capacidades se desarrollan en el tiempo, es decir, son producto de un proceso de crecimiento que la persona experimenta en el transcurso de su vida, no solo en su etapa escolar, donde intervienen aspectos relacionados tanto con la adquisición de conocimientos (procesos de aprendizaje) como con la ejecución de tareas.

Además es importante tener en cuenta para el análisis de estudios posteriores que, si bien es cierto, existe variedad de definiciones del término "competencias", así como elementos comunes que las identifican, se han mencionado problemas en ellas debido a su reduccionismo o falta de especificidad con otros conceptos (Tobón, 2006) y a la forma en la que normalmente se enfocan los aspectos tangibles e intangibles relacionados con ellas (Tobón, 2013).

No obstante, indistintamente de la definición que se adopte, las competencias involucran el desarrollo de habilidades y capacidades en las personas que le permiten interactuar con su entorno, lo cual puede ser aprovechado en cualquier asignatura del currículo escolar, en especial en aquellas que buscan explorar las posibles aplicaciones de los saberes en un determinado contexto.

\section{Tipos de competencias}

En términos generales, en la literatura se plantean dos tipos de competencias: las que se relacionan con un área o campo de estudio, denominadas "competencias específicas", referidas a un campo de conocimiento particular, y las competencias generales, que son comunes a todos los campos.

\section{Competencias generales}

El proyecto Tuning plantea que las competencias genéricas incluyen un conjunto de aspectos compartidos o comunes en cualquier campo de estudio, entre las que se consideran las habilidades personales, la capacidad de aprender, de tomar decisiones, de diseñar proyectos, etc. (Beneitone, Esquetini, Gonzáles, Maletá, Siufi y Wagenaar, 2007). Es decir, las competencias generales incluyen aspectos cognitivos, emocionales, sociales, ambientales, entre otros, que se combinan al momento de realizar una serie de actividades con el fin de resolver una tarea particular. Estas competencias permiten 
el logro de un aprendizaje integrado en el cual contenido, habilidad y capacidad se unen con el fin de resolver situaciones inéditas (Díaz, 2006).

\section{Competencias específicas}

En cuanto a las competencias específicas, el proyecto Tuning menciona que estas hacen referencia a las habilidades, destrezas y conocimientos específicos que se espera sean desarrolladas por una persona en su área de estudio (González y Wagenaar, 2003). El desarrollo del pensamiento disciplinar es un proceso más complejo que no solo involucra la adquisición de conocimientos que demande únicamente la aplicación de procesos de memorización de información, también incluye el desarrollo de actividades que requieran generar comprensión, explicación o incluso traducción de la temática al lenguaje de cada una de las personas participantes en el proceso (Díaz, 2006).

\section{El enfoque por competencias}

De acuerdo con Mulder, Weigel y Collins (2007) en el enfoque por competencias destacan tres tradiciones:

- La conductista, que destaca la importancia de observar a trabajadores que se consideran exitosos y efectivos, con la intención de precisar qué es lo que les otorga esas cualidades.

- La genérica, que pretende identificar las habilidades comunes que explican por qué existen diferencias en el desempeño.

- La cognitiva, que involucra los recursos mentales que usan las personas para hacer los trabajos importantes, adquirir conocimientos y obtener un buen desempeño en la tarea que realizan.

Dado que existen muchas conceptualizaciones del término "competencias", se tomará como base la propuesta por el proyecto Tuning debido a que por una parte se centra en la estructura y contenido de los planes de estudio a nivel de educación superior, y por otra se orienta sobre todo en las competencias genéricas y específicas.

De acuerdo con la definición dada por el proyecto Tuning, las competencias son capacidades desarrolladas por la persona producto de su proceso de aprendizaje, de modo que le permiten alcanzar la habilidad para desenvolverse en un contexto particular. En el caso del ámbito científico, la OCDE (2017) busca promover las competencias en diferentes ámbitos de la ciencia bajo el contexto de la "alfabetización científica", la cual se entiende como la capacidad de la persona para involucrarse en temas relacionados con el conocimiento científico.

Diferentes propuestas se han generado sobre metodologías de enseñanza que pretenden mejorar la labor docente de modo que los objetivos de aprendizaje que se han propuesto en los planes de estudio sean alcanzados por parte del estudiantado. 
Entre las metodologías propuestas está la enseñanza por competencias, sobre la cual se ha presentado una serie de argumentos que respaldan su incorporación en los planes de estudios de las instituciones educativas, dado que los procesos educativos tienen la tarea de promover el desarrollo de estas competencias.

En primer lugar, las constantes transformaciones culturales, sociales y tecnológicas en la sociedad ofrecen consigo oportunidades de integración e innovación en los distintos procesos de la actividad humana. Con el fin de responder adecuadamente a tales transformaciones, la educación superior ha dirigido sus acciones a la adopción del enfoque por competencias (Arias y Lombillo, 2019).

En segundo lugar, la enseñanza por competencias se presenta como una opción alternativa en el campo de la educación con la promesa de que su implementación puede llevar a realizar mejores procesos de formación académica (Díaz, 2006). Como tercer argumento se ha considerado que la incorporación de currículos basados en competencias hace que el aprendizaje sea más activo, ya que considera como centro del proceso a la persona, dada su orientación a la vinculación entre teoría y práctica por medio de la integración de conocimientos fundamentales (Casanova, Canquiz, Paredes e Inciarte, 2018).

De acuerdo con lo anterior, la enseñanza por competencias está orientada a promover en la persona el desarrollo de habilidades que le permitan hacerle frente a una situación particular, en la que teoría y práctica se combinan con el fin de establecer las acciones más adecuadas. Además la educación debe trascender del énfasis en conocimientos a un enfoque más integral en el que intervienen aspectos como el afecto, la motivación, el interés y la responsabilidad de las personas en formación (Casanova et al., 2018).

Desde esta perspectiva, la enseñanza por competencias propicia la formación de personas capaces de resolver problemas que se harán presentes en los diferentes entornos de la actividad humana. Para ello, se considera la adquisición de conocimientos como parte importante del proceso, pero no constituye el aspecto fundamental, es decir, lo que se persigue es que los conocimientos se utilicen en la búsqueda de soluciones, pero estos no deben ser el fin primordial de la acción educativa.

En este particular, un enfoque educativo por competencias permite promover la resolución de problemas que emergen de la vida cotidiana; sin embargo, es necesaria para ello la articulación entre diferentes elementos como conocimientos, capacidades, actitudes o acciones, lo que hace complejo este proceso, por su carácter multidimensional.

Por ejemplo, en el contexto costarricense esto se evidencia en los procesos de transformación llevados a cabo por la Escuela de Matemática de la Universidad Nacional que ha implementado, desde el año 2017, un nuevo plan de estudios en la carrera Bachillerato y Licenciatura en la Enseñanza de la Matemática basado en el 
enfoque por competencias, como una forma de enfrentar los cambios provocados por los diversos fenómenos que han influenciado el estilo de vida de las sociedades.

Como parte de este plan, se han definido competencias tanto generales como específicas, considerándose para estas últimas tres áreas: matemáticas, didáctico-matemáticas y pedagógicas. No obstante, desde su implementación existe la consciencia y necesidad de revisión sobre la estructura y saberes que se desarrollan.

$\mathrm{Al}$ respecto el programa de estudios, en el área disciplinar de Estadística, ofrece tres cursos: Estadística y Probabilidad, Inferencia Estadística y Didáctica de la Estadística y la Probabilidad, donde se incorporan tanto competencias generales como específicas, pero surge la interrogante de si las competencias matemáticas establecidas en el plan son apropiadas para abordar las temáticas de los cursos del área de estadística o si, por el contrario, será necesaria la incorporación de competencias más adecuadas a la naturaleza de la disciplina.

En la opinión del equipo investigador, es importante considerar el segundo planteamiento. Esta posición se sustenta en las particularidades del campo específico de conocimiento, diferente al de la matemática y a las recomendaciones que la educación estadística brinda para su enseñanza, especialmente a nivel universitario.

En el siguiente apartado se detallará de manera más precisa cuáles son las principales diferencias entre ambas disciplinas y cómo el enfoque por competencias se ajusta con los principios y las buenas prácticas educativas propuestas por la educación estadística.

\section{Competencias y educación estadística}

El término de "educación estadística" empezó a utilizarse por el año de 1973 cuando la Asociación Americana de Estadística (ASA por sus siglas en inglés) denominó Sección de Educación Estadística (Ben-Zvi y Garfield, 2008) a lo que en el año 1944 había denominado como Sección de Capacitación de Estadísticos (Mason, Mckenzie y Ruberg, 1990).

El enfoque inicial de la estadística era la formación de personas especialistas en la disciplina, sin embargo, con el tiempo se amplió para incluir la formación en todos los niveles, por ejemplo, en la década de 1960 nació el interés en el campo de la educación matemática por enseñar a las personas de nivel preuniversitario sobre el uso y análisis de datos, mientras que para los años 1980 se planteó que la estadística debía incorporarse en la educación preuniversitaria y mejorarse en la educación postsecundaria, y para la década de 1990 la discusión se centró en que la educación estadística debía enfocarse en la alfabetización, el razonamiento y el pensamiento estadísticos (Ben-Zvi y Garfield, 2008).

Sin embargo, la inclusión de la estadística en los planes de estudios de los diferentes niveles educativos vino a plantear una serie de discusiones sobre la forma en 
que los cursos de esta disciplina debían abordarse. Por ejemplo, Moore (1997) mencionaba sugerencias para la revisión de la metodología de enseñanza en los cursos introductorios de estadística para pasar a un modelo constructivista de aprendizaje, en el que la persona docente guíe a sus estudiantes hacia unas competencias y conocimientos estadísticos más específicos.

Por otro lado, en la economía del siglo XXI, caracterizada por un mercado laboral en constante cambio, con rápida rotación de personal y necesitado de mano de obra calificada con conocimientos matemáticos y estadísticos para su buen desempeño, es importante una educación estadística que fortalezca la comprensión de las estadísticas y la interpretación de los datos. Estas habilidades se consideran necesarias para la toma de decisiones, tanto individuales como sociales, fundamentadas en datos (Legaki y Hamari, 2020).

Por otra parte, la gran cantidad de información de la que se dispone actualmente provoca que el uso de herramientas tecnológicas adquiera mayor relevancia, lo que lleva consigo un replanteamiento del enfoque de la educación estadística. Es así como la incorporación de temas de estadística en los planes de estudio de la educación primaria y secundaria en diferentes partes del mundo puede verse como una respuesta a la necesidad de fomentar el desarrollo de capacidades de las personas para el manejo y análisis de la información.

La enseñanza de la estadística en los sistemas educativos ha sido un tema de gran interés en los años recientes (Batanero, Burrill y Reading, 2011); asimismo el desarrollo de investigaciones en el área ha proporcionado una variedad de recursos con el fin de contribuir con la labor de aula de las personas encargadas de impartir los cursos de Estadística. Estos esfuerzos por integrar en la práctica docente los diferentes aportes que los procesos de investigación han generado se orientan en la idea de mejorar la experiencia educativa del estudiantado que accede a los cursos de esta disciplina.

Por consiguiente, la educación estadística puede considerarse como una disciplina emergente, que surge a partir la estadística y la matemática y que actualmente se establece como un campo de estudio propio, que centra su papel en el desarrollo de habilidades y la mejora de la educación en todos los niveles (Ben-Zvi y Garfield, 2008).

\section{Competencia matemática}

En cuanto a las competencias y su relación con la educación estadística, es evidente que su análisis pasa por una categorización de competencias disciplinares. En este apartado se abordan las correspondientes del área de matemática, dado que en la mayoría de los programas escolares la estadística está incorporada como un tema más del currículo de Matemática. A pesar de ello, esto no implica una equivalencia entre competencias matemáticas y estadísticas, como se detallará más adelante. 
En cuanto a la conceptualización del término, el proyecto PISA define la competencia matemática como "la capacidad de un individuo para identificar y comprender el papel que las matemáticas juegan en el mundo, realizar razonamientos bien fundados y utilizar e involucrarse en las matemáticas de manera que satisfaga las necesidades de la vida del individuo como ciudadano constructivo, comprometido y reflexivo" (INCE 2004, p. 12). Asimismo, Niss (2003) define competencia matemática como la "habilidad para comprender, juzgar, hacer y usar las matemáticas en una variedad de contextos intra y extra matemáticos” (p. 218).

Por su parte, Jiménez (2010) considera la competencia probabilística como parte de la competencia matemática y menciona que "el alumno es capaz de implementar métodos para recolectar, sistematizar y analizar diferentes tipos de datos, comprendiendo el fenómeno (económico, político, social, profesional, entre otros) y proponiendo soluciones razonables a problemas en los que no hay una solución clara y segura, abordándolos con un espíritu crítico de investigación” (p. 7).

De las definiciones anteriores se desprende la relación que deberían tener los conocimientos matemáticos con las aplicaciones en la vida cotidiana de las personas, es decir, la idea es que las personas desarrollen capacidades que les permitan apreciar la utilidad de la matemática como herramienta para resolver problemas. También es interesante observar que se hace referencia a la utilidad de la matemática de acuerdo con el contexto, el cual no necesariamente debería tener una aplicación inmediata o relacionada con la vida de las personas. Este último punto es una de las diferencias fundamentales entre competencias matemáticas y estadísticas, pues a diferencia de las primeras, las segundas surgen de manera natural del contexto.

Por ejemplo, PISA no diferencia entre competencias matemáticas y estadísticas, no obstante, a pesar de la estrecha relación entre Matemática y Estadística en los currículos escolares, hay autores que señalan que esta última disciplina no es una rama de la matemática (delMas, 2004; Moore, 1988; Rossman, Chance, Medina y Obispo, 2006). Entre las principales diferencias de la estadística con respecto a la matemática se destacan:

- Los datos cobran sentido en el momento en que se asocian con el contexto de donde provienen.

- Las circunstancias en las que se lleva a cabo la recolección de los datos son de suma importancia.

- La variabilidad es un tema central.

- Es inevitable tener que lidiar con la incertidumbre en los resultados.

Asimismo, la matemática no debe tener necesariamente una utilidad práctica inmediata, pues muchas veces se generan teorías en esta disciplina que hasta tiempo después sirven de base para aplicaciones en un campo específico. En contraste, la gran mayoría de los conocimientos y procedimientos desarrollados en la estadística 
son usados para resolver problemas prácticos, dado su carácter multidisciplinario que permea en distintos campos del conocimiento.

Como puede apreciarse, la naturaleza de ambas disciplinas es distinta y, aunque compartan elementos en común, en este contexto la matemática es usada por la estadística más como herramienta que como un fin en sí mismo, por lo tanto, esa diferencia debería verse reflejada en la forma en que se plantean las competencias en un programa de estudios, pues cuando se enseña estadística el contexto se constituye en el centro de toda actividad (Gal, 2019). Es decir, resulta necesario plantear competencias estadísticas que respondan a las características propias de la disciplina, pues las competencias matemáticas no son suficientes para desarrollar las habilidades, conductas y destrezas que se requieren.

\section{Competencia estadística}

Como ya se ha mencionado, aunque existe relación entre las competencias matemáticas y las competencias estadísticas, no son lo mismo. Si bien es cierto, la competencia matemática está relacionada con el rendimiento en cursos de Estadística (Johnson y Kuennen, 2006; Lalonde y Gardner, 1993; Wisenbaker, Scott y Nasser, 2000), la matemática y la estadística son dos disciplinas metodológicas diferentes (Gal y Garfield, 1997; Groth, 2007; Moore, 1988).

A manera de ejemplo, en el contexto de un programa de posgrado en Ciencias Pedagógicas, Gorina-Sánchez y Alonso-Berenguer (2013) definen competencia estadística como:

[...] la posibilidad de reconocer, en cada etapa de la investigación pedagógica, cuándo se necesita hacer uso de la Estadística, teniendo conciencia de la variedad de interpretaciones posibles de los resultados al procesar los datos para sustentar o rechazar un argumento, siendo capaz de formular hipótesis estadísticas, seleccionar y aplicar la técnica apropiada para la recolección y procesamiento de los datos, verificar los supuestos de las técnicas que se empleen, seleccionar adecuadamente los estimadores y niveles de precisión, emplear apropiadamente ciertos software estadísticos existentes, así como explicar la lógica de los procesos estadísticos empleados y los resultados alcanzados, con la suficiente honradez científica [p. 152].

Con respecto al tema de las competencias en estadística, varios autores concuerdan en que la enseñanza debe centrarse en desarrollar principalmente tres: la alfabetización estadística, el razonamiento estadístico y el pensamiento estadístico (Chance, 2002; Gal, 2002; Garfield, 2002; Pfannkuch y Wild, 2004). Uno de los principales argumentos a favor plantea que los enfoques tradicionales para la enseñanza de la estadística centrados en habilidades, procedimientos y cálculos no llevan a los estudiantes a razonar o pensar estadísticamente (Ben-Zvi y Garfield, 2008).

Por lo tanto, se considera relevante comprender el significado de estas tres competencias. En lo que concierne a la alfabetización estadística, esta se refiere a 
la capacidad de las personas para interpretar y evaluar críticamente la información estadística, los argumentos relacionados con los datos o los fenómenos estocásticos, que pueden encontrar en diversos contextos, y cuando sea relevante, y su capacidad para discutir o comunicar sus reacciones a dicha información estadística, como su comprensión del significado de la información, sus opiniones sobre las implicaciones de esta información o sus preocupaciones con respecto a la aceptabilidad de conclusiones (Gal, 2002).

En consecuencia, la alfabetización estadística incluye habilidades básicas e importantes que pueden usarse para comprender información estadística o resultados de investigación (capacidad de organizar datos, construir y mostrar tablas y trabajar con diferentes representaciones de datos), también incluye la comprensión de conceptos, vocabulario y símbolos, así como una comprensión de la probabilidad como medida de incertidumbre (Ben-Zvi y Garfield, 2004).

Por otra parte, el razonamiento estadístico puede considerarse como la manera en que las personas utilizan las ideas para dar sentido a la información estadística, lo cual requiere hacer interpretaciones basadas en un conjunto de datos, representaciones gráficas y resúmenes estadísticos que involucran establecer relaciones con otros conceptos como el de distribución, dispersión, muestreo, asociación, incertidumbre y aleatoriedad (Garfield, 2002).

Finalmente, el pensamiento estadístico se define como una serie de procesos de pensamiento que reconocen que la variación está a nuestro alrededor y presente en todo lo que hacemos, todo el trabajo es una serie de procesos interconectados, y la identificación, caracterización, cuantificación, control y reducción de la variación brinda oportunidades de mejora (Snee, 1991). Por otro lado, según Moore (1997), el pensamiento estadístico incluye los siguientes elementos: la necesidad de datos, la importancia de la producción de datos, la omnipresencia de variabilidad, la medición y modelización de la variabilidad.

Además, algunos de los elementos del pensamiento estadístico involucrados en una investigación basada en datos pueden organizarse en un marco de cuatro dimensiones: un ciclo investigador, el tipo de pensamiento, un ciclo interrogativo y las disposiciones (Wild y Pfannkuch, 1999).

El ciclo investigador se refiere a la forma en que se actúa y en lo que se piensa durante el desarrollo de una investigación estadística. El tipo de pensamiento involucra tipos generales como el estratégico, busca explicaciones, modelización y aplicación de técnicas, así como elementos centrales del pensamiento estadístico: reconocimiento de la necesidad de los datos, transnumeración, percepción de la variación, razonamiento con modelos estadísticos y la integración de la estadística con el contexto.

El ciclo interrogativo es un proceso de pensamiento genérico en uso constante en la resolución de problemas estadísticos que involucra actividades como generar, 
buscar, interpretar, juzgar y criticar. Las disposiciones involucran las cualidades personales (curiosidad, perseverancia, compromiso, imaginación, escepticismo, ser lógico) que afectan el ingreso en un modo de pensamiento.

La tabla 1 muestra un resumen con las tareas que involucran cada una de lo que se ha considerado en este documento como competencias estadísticas.

Tabla 1. Tareas que caracterizan cada competencia estadística.

\begin{tabular}{ccc}
\hline Alfabetización & Razonamiento & Pensamiento \\
\hline Identificar & ¿Por qué? & Aplicar \\
Describir & ¿Cómo? & Criticar \\
Traducir & Explicar & Evaluar \\
Interpretar & & Generalizar \\
Leer & & \\
\hline
\end{tabular}

Fuente: delMas (2002, p. 6).

En resumen, la incorporación de estas tres competencias en las asignaturas del área de estadística a lo largo del tiempo proporcionará a las personas, tanto en su etapa escolar como en su vida adulta, desarrollar habilidades y capacidades que les permitan entender, interpretar, representar, aplicar y eventualmente cuestionar información que contenga datos estadísticos. Como resulta evidente, no es posible plantear estas competencias desde el área de la matemática, aunque esto no limita que pueden ser complementadas con otras competencias que sí corresponden a este campo de conocimiento.

\section{ConClusión}

La enseñanza por competencias constituye un enfoque educativo que ha sido aceptado e implementado en los currículos escolares por varios países, lo que le da sustento, y aunque pueden existir críticas a sus fundamentos e ideas, llevado a la práctica de modo apropiado tiene el potencial para desarrollar las habilidades y capacidades de las personas, contribuyendo así al mejoramiento de los procesos educativos.

Por otro lado, la educación estadística como disciplina emergente busca mejorar la adquisición de conocimientos disciplinares con el objetivo de que las personas puedan no solo conocer sobre el lenguaje estadístico, sino además comprender conceptos relevantes que les permitan tomar decisiones informadas sobre los datos que leen y analizan.

Para ello se necesita de la integración de metodologías de enseñanza eficaces que se adapten a los requerimientos de una sociedad que demanda una ciudadanía más consciente sobre el tratamiento y análisis de información. El enfoque por competencias promueve la aplicación de metodologías de trabajo que pueden contribuir 
a los propósitos de la educación estadística, puesto que las ideas desarrolladas en la investigación ponen de manifiesto la posibilidad de su implementación.

Sin embargo, es importante considerar que el abordaje de un programa educativo para el desarrollo de competencias podría implicar romper con la estructura tradicional de un currículo basado en contenidos y promover el uso de situaciones problema enmarcadas en un contexto real que le permitan a la persona el desarrollo de habilidades de pensamiento y razonamiento estadísticos que favorecen la comprensión y aplicación de los conceptos fundamentales de la estadística.

Asimismo, una de las tareas más importante en este aspecto es cómo desarrollarlas en el estudiantado. Algunas acciones que se han mencionado al respecto para ser incorporadas en las clases son: (a) trabajar con datos reales relacionados con el contexto en el cual el estudiantado está inmerso y (b) incentivar al estudiantado a interpretar, explicar, criticar, justificar y evaluar los resultados, preferiblemente en forma grupal, discutiendo e intercambiando opiniones (Campos, 2007).

Por lo tanto, la educación estadística aspira a promover en las personas habilidades y capacidades referentes al manejo de datos que podrían resumirse en fomentar la alfabetización, el razonamiento y el pensamiento estadístico, las cuales podrían considerarse como competencias disciplinares o específicas que pueden ser promovidas mediante metodologías afines al enfoque que plantea la enseñanza por competencias.

Por ejemplo, para desarrollar la alfabetización estadística en el estudiantado, el personal docente puede pedirles que identifiquen ejemplos o instancias de un término o concepto, describan gráficos, distribuciones y relaciones, reformulen o traduzcan hallazgos estadísticos o interpreten los resultados de un procedimiento estadístico (delMas, 2002). También es importante tomar en cuenta que para tener éxito en el proceso de alfabetización estadística es necesario estimular el desarrollo de habilidades básicas de lectura y comprensión así como otras de más alto nivel como la interpretación, predicción y pensamiento crítico, las cuales deben ser enseñadas para crear personas informadas, dado que estas habilidades no surgen de manera natural (Sharma, 2017).

Asimismo, para promover el razonamiento estadístico las tareas deben orientarse a que expliquen por qué o cómo se produjeron los resultados (por ejemplo, explicar el proceso que produce la distribución de muestreo de una estadística, explicar cómo la media actúa como un punto de equilibrio, explicar por qué la mediana no es sensible a los valores extremos, o explicar por qué una muestra aleatoria tiende a producir una muestra representativa) o por qué una conclusión está justificada (delMas, 2002).

Además, algunas técnicas que permiten evaluarlo son: (a) estudios de casos o tareas auténticas, (b) mapas conceptuales, (c) críticas de ideas o problemas estadísticos en las noticias, (d) documentos de minutas y (e) elementos de opción múltiple mejorados (Garfield y Chance, 2000). 
En cuanto al pensamiento estadístico, su desarrollo puede promoverse cuando en el proceso educativo se desafía al estudiantado a aplicar su comprensión a los problemas del mundo real, a criticar y evaluar el diseño y las conclusiones de los estudios, o a generalizar el conocimiento obtenido de los ejemplos del aula a situaciones nuevas y algo novedosas (delMas, 2002).

Finalmente, es importante señalar las recomendaciones emanadas de la Guidelines for Assessment and Instruction in Statistics Education (GAISE), en su informe referido a educación superior (GAISE, 2016), el cual manifiesta la importancia de enfatizar en el pensamiento estadístico. Las seis recomendaciones de la GAISE para el profesorado que imparte cursos de Estadística son: promover el aprendizaje activo, enseñar estadística como un proceso de resolución de problemas y toma de decisiones, centrarse en entender los conceptos, integrar datos reales con un contexto y propósito, usar tecnología para explorar conceptos y analizar datos, usar evaluaciones que mejoren y evalúen el aprendizaje del estudiantado (GAISE, 2016).

Como puede apreciarse, dichas recomendaciones pueden incorporarse en un currículo basado por competencias, ya que ambos enfoques se complementan. No obstante, también es evidente que las competencias necesarias para lograr este cometido no son las que tradicionalmente se enmarcan en el área de la matemática, por lo que se requieren competencias específicas del área de la estadística, diferentes a las propuestas en matemática.

\section{RECONOCIMIENTO}

Este trabajo fue elaborado en el marco del proyecto de investigación "Una propuesta didáctica, desde el enfoque por competencias, para la enseñanza de la Estadística y Probabilidad en la carrera de Bachillerato y Licenciatura en Enseñanza de la Matemática de la Universidad Nacional”, código SIA 0064-19, Universidad Nacional, Costa Rica.

\section{REFERENCIAS}

Arias, C. G., y Lombillo, I. (2019). Reflexiones en torno al enfoque de formación basado en competencias en el contexto chileno. Revista Cubana de Educación Superior, 38(3). Recuperado de: http:/ / scielo.sld.cu/pdf/ rces/v38n3/0257-4314-rces-38-03-e19.pdf.

Batanero, C., Burrill, G., y Reading, C. (2011). Teaching statistics in school mathematics: Challenges for teaching and teacher education. A joint ICMI/LASE study. The18th ICMI study. Heidelberg: Springer. Recuperado de: http://ndl. ethernet.edu.et/bitstream/123456789/33856/1/420. Carmen\%20Batanero.pdf.
Beneitone, P., Esquetini, C., Gonzáles, J., Maletá, M. M., Siufi, G., y Wagenaar, R. (eds.) (2007). Reflexiones y perspectivas de la educación superior en América latina (Informe Final-Proyecto Tuning-América Latina 2004-2007). Recuperado de: http://tuning.unideusto.org/tuningal/index. phpoption $=$ com_docman\&Itemid $=191 \&$ task $=$ view_ c a tegory \& catid $=22 \&$ order $=$ d mdate_ published \&ascdesc $=$ DESC.

Ben-Zvi, D., y Garfield, J. (2008). Introducing the emerging discipline of statistics education. School Science and Mathematics, 108(8), 355-361. Recuperado de: https:// 
scholar.google.es / scholar?cluster $=10537554806932$ 61370\&hl=es\&as_sdt $=0,5$.

Ben-Zvi, D., y Garfield, J. (2004). Statistical literacy, reasoning, and thinking: Goals, definitions, and challenges. En D. Ben-Zvi y J. Garfield (eds.), The challenge of developing statistical literacy, reasoning and thinking (pp. 3-15). Dordrecht, Países Bajos: Kluwer Academic Publisher. Recuperado de: https://www. researchgate.net/publication/224013565_Towards_ an_Understanding_of_Statistical_Thinking.

Campos, C. R. (2007). A educação estatística: uma investigação acerca dos aspectos relevantes à didática da estatística em cursos de graduação [Tesis Doctoral]. UNESPIGCE, Rio Claro. Recuperado de: http://hdl.handle. net/11449/102161.

Casanova, I., Canquiz, L., Paredes, I., e Inciarte, A. (2018). Visión general del enfoque por competencias en Latinoamérica. Revista de Ciencias Sociales, 24(4), 114-125. Recuperado de: https://www.redalyc.org/ jatsRepo/280/28059581009/html/index.html.

Chance, B. (2002). Components of statistical thinking and implications for instruction and assessment. Journal of Statistics Education, 10(3). https:/ / doi.org/ 10.1080/10691898.2002.11910677.

De Allende, C., y Morones, G. (2006), Glosario de términos vinculados con la cooperación académica. México: ANUIES. Recuperado de: http://www.cucs. udg.mx/avisos/PDC_19_02_08/glosario_terminos_vinculados_con_cooperacion_acad.pdf.

delMas, R. (2002). Statistical literacy, reasoning, and learning: a commentary. Journal of Statistics Education, 10(3). https://doi.org/10.1080/10691898.2002.119 10679.

delMas, R. (2004). A comparison of mathematical and statistical reasoning. En D. Ben-Zvi y J. Garfield (eds.), The challenge of developing statistical literacy, reasoning and thinking (pp. 79-95). Dordrecht: Springer. Recuperado de: https://www.researchgate.net/ publication/224013565_Towards_an_Understanding_of_Statistical_Thinking.

Díaz, Á. (2006). El enfoque de competencias en la educación. ¿Una alternativa o un disfraz de cambio? Perfiles Educativos, 28(111), 7-36. Recuperado de: https:// www.redalyc.org/articulo.oa?id=132/13211102.
Friz, M., Sanhueza, S., y Figueroa, E. (2011). Concepciones de los estudiantes para profesor de matemáticas sobre las competencias profesionales implicadas en la enseñanza de la estadística. Revista Electrónica de Investigación Educativa, 13(2), 113-131. Recuperado de: http:// redie. uabc.mx/vol13no2/contenido-frizsanhueza.html.

GAISE (2016). Guidelines for Assessment and Instruction in Statistics Education. College report. Alexandria, VA: American Statistical Association. Recuperado de: www.amstat. org/asa/files/pdfs/GAISE/GaiseCollege_Full.pdf.

Gal, I. (2002). Adults' statistical literacy: meanings, components, responsibilities. International Statiscal Review, 70(1), 1-51. Recuperado de: https://iase-web.org/ documents/intstatreview/02.

Gal, I. (2019). Understanding statistical literacy: About knowledge of contexts and models. En J. M. Contreras, M. M. Gea, M. M. López-Martín y E. Molina-Portillo (eds.), Actas del Tercer Congreso Internacional Virtual de Educación Estadística. Recuperado de: www.ugr.es/local/ fqm126/civeest.html.

Gal, I., y Garfield, J. (1997). Curricular goals and assessment challenges in statistics education. En I. Gal y J. B. Garfield (eds.), The assessment challenge in statistics education (pp. 1-13). IOS Press. Recuperado de: https://www.stat.auckland.ac.nz/ iase/publications/ assessbk/chapter01.pdf.

Garfield, J. (2002). The challenge of developing statistical reasoning. Journal of Statistics Education, 10(3). https:/ / doi.org/10.1080/10691898.2002.11910676.

Garfield, J., y Chance, B. (2000). Assessment in Statistics education: Issues and challenges. Mathematics thinking and learning, 2, 99-125. https://doi.org/10.1207/ S15327833MTL0202_5.

González, J., y Wagenaar, R. (2003). Tuning educational structures in Europe. Informe Final Fase I. Bilbao, España: Universidad de Deusto. Recuperado de: http:// tuningacademy.org/wp-content/uploads/2014/02/ TuningEUI_Final-Report_SP.pdf.

Gorina-Sánchez, A., y Alonso-Berenguer, I. (2013). Concepción de una competencia estadística para el estudiante de doctorado en Ciencias Pedagógicas. Probabilidad condicionada: Revista de Didáctica de la Estadística, 1,149-156. Recuperado de: https://dialnet.unirioja.es/ descarga/articulo/4770258.pdf. 
Groth, R. E. (2007). Toward a conceptualization of statistical knowledge for teaching. Journal for Research in Mathematics Education, 38(5), 427-437. Recuperado de: http://www.jstor.com/stable/30034960.

INCE (2004). Marcos teóricos de PISA 2003: la medida de conocimientos y destrezas en matemáticas, lectura, ciencias y resolución de problemas. Madrid: MEC. Recuperado de: www.ince.mec.es/pub/marcoteoricopisa2003.pdf.

Jiménez, M. (2010). Competencias en matemáticas y estadística. Recuperado de: http://www.inacap.org/tportal/portales/tp4964b0e1bk102/uploadImg/File/competenciasMatematicasEstadistica.pdf.

Johnson, M., y Kuennen, E. (2006). Basic math skills and performance in an introductory statistics course. Journal of Statistics Education, 14(2). Recuperado de: http://jse. amstat.org/v14n2/johnson.html.

Lalonde, R. N., y Gardner, R. C. (1993). Statistics as a second language? A model for predicting performance in psychology students. Canadian Journal of Behavioural Science/Revue Canadienne des Sciences du Comportement, 25(1), 108. https://doi.org/10.1037/h0078792.

Legaki, Z., y Hamari, J. (2020). Gamification in statistics education: A literature review. En International GamiFIN Conference (pp. 41-51). CEUR-WS. Recuperado de: http://ceur-ws.org/Vol-2637/paper5.pdf.

Mason, R. L., McKenzie, J. D. Jr., y Ruberg, S. J. (1990). A brief history of the American Statistical Association, 1839-1989. American Statistician, 44(2), 68-73. Recuperado de: https://www.jstor.org/stable/2684130.

Mejía, W. (2010). El enfoque por competencias. Recuperado de: http:/ / estadisticaestudiante.pbworks.com/w/file/ fetch/75516812/EL\%20ENFOQUE $\% 20 D E \% 20$ COMPETENCIAS\%201.pdf.

Moore, D. S. (1997). New pedagogy and new content: The case of statistics. International Statistical Review, 65(2), 123-137. Recuperado de: http://iase-web.org/documents/intstatreview/97.Moore.pdf.

Moore, D. S. (1988). Should mathematicians teach statistics? College Mathematics Journal, 19(1), 3-7. https:/ / doi. org/10.1080/07468342.1988.11973073.

Mulder, M., Weigel, T., y Collins, K. (2007). The concept of competence in the development of vocational education and training in selected EU member states: A critical analysis. Journal of Vocational Education \& Training, 59(1), 67-88. https://doi.org/10.1080/13636820601145630.
Niss, M. (2003). Quantitative literacy and mathematics competencies. En Quantitative literacy: Why numeracy matters for schools and colleges (pp. 215-220). Recuperado de: https://rieoei.org/historico/ deloslectores/6761Iniguez.pdf.

OCDE (2004). El Programa PISA de la OCDE. Qué es y para qué sirve. París: OCDE. Recuperado de http:// www.oecd.org/pisa/39730818.pdf.

OECD (2017). PISA 2015 assessment and analytical framework: Science, reading, mathematic, financial literacy and collaborative problem solving. Revised edition. París: PISA/ OECD Publishing. http://dx.doi. org/10.1787/9789264281820-en.

Perrenoud, P. (2004). Diez. nuevas competencias para enseñar. México: SEP. Recuperado de: https://www.uv.mx/ dgdaie/files/2013/09/Philippe-Perrenoud-Dieznuevas-competencias-para-ensenar.pdf.

Pfannkuch, M., y Wild, C. (2004). Towards and understanding of statistical thinking. En D. Ben-Zvi y J. Garfield (eds.), The challenge of developing statistical literacy, reasoning and thinking (pp. 17-46). Dordrecht, Países Bajos: Kluwer Academic Publisher. Recuperado de: https:/ / www.researchgate.net/publication/224013565_ Towards_an_Understanding_of_Statistical_Thinking. Rossman, A., Chance, B., Medina, E., y Obispo, C. P. S. L. (2006). Some key comparisons between statistics and mathematics, and why teachers should care. En Thinking and reasoning with data and chance: Sixty-eighth annual yearbook of the National Council of Teachers of Mathematics (pp. 323-333). Recuperado de https:// statweb.calpoly.edu/arossman/stat400/Rossman_ Chance_Medina.pdf.

Sharma, S. (2017) Definitions and models of statistical literacy: a literature review. Open Review of Educational Research, 4(1), 118-133, https://doi.org/10.1080/23 265507.2017.1354313.

Snee, R. (1991). Statistical thinking and its contribution to total quality. 34th Fall Technical Conference. Recuperado de: http://asq.org/statistics/2011/10/continuousimprovement/statistical-thinking-and-its-contribution-to-total-quality.pdf.

Tobón, S. (2006). Aspectos básicos de la formación basada en competencias. Talca: Proyecto Mesesup. Recuperado de: https://maristas.org.mx/gestion/web/doctos/ aspectos_basicos_formacion_competencias.pdf. 
Tobón, S. (2013). Formación integral y competencias. Pensamiento complejo, currículo, didáctica y evaluación. Bogotá: ECOE. Recuperado de: https://www.researchgate. net/profile/Sergio_Tobon4/publication/319310793_ Formacion_integral_y_competencias_Pensamiento_complejo_curriculo_didactica_y_evaluacion / links/59a2edd9a6fdcc1a315f565d/Formacion-integraly-competencias-Pensamiento-complejo-curriculodidactica-y-evaluacion.pdf.

Wild, C. J., y Pfannkuch, M. (1999). Statistical thinking in empirical enquiry (with discussion). International Statistical
Review, 67(3), 223-265. Recuperado de: https:// iase-web.org/documents/intstatreview/99.Wild. Pfannkuch.pdf.

Wisenbaker, J. M., Scott, J. S., y Nasser, F. (2000). Structural equation models relating attitudes about and achievement in introductory statistics courses: A comparison of results from the US and Israel. En Ninth International Congress on Mathematics Education, Tokyo. Recuperado de: http://iase-web.org/documents/papers/icme9/ ICME9_01.pdf.

Cómo citar este artículo:

Aguilar Fernández, E., Zamora Araya, J. A., y Guillén Oviedo, H. S. (2021). Alfabetización, razonamiento y pensamiento estadísticos: competencias específicas que requieren promoverse en el aula. IE Revista de Investigación Educativa de la REDIECH, 12, e1118. doi: doi.org/10.33010/ie_rie_rediech.v12i0.1118. 